\title{
Maximum Strength in Squats Determines Jumping Height in Young Female Volleyball Players
}

\author{
Sofia Ryman Augustsson* \\ Department of Food and Nutrition and Sport Science, Faculty of Education, University of Gothenburg, Box 300, SE \\ 40530 Göteborg, Sweden
}

\begin{abstract}
Few data exist on the relationship between maximum strength and power performance, such as jumping ability in low-level female volleyball players. The aim of the present study was to determine the relationship between maximum strength and jumping performance in young female non-elite volleyball players before and after an in-season resistancetraining protocol. A 26-week, in-season resistance-training program was carried out on ten female volleyball players, aged $19( \pm 2)$. The 1 RM squat test was used to evaluate the players' maximum strength in the lower extremities and a vertical jump (VJ) test was used to assess functional performance and power. There was a significant enhancement of $69 \%$ $(p=0.005)$ for the squat test and $9 \%$ for the VJ test $(p=0.008)$. A strong significant relationship was found between the VJ test and the $1 \mathrm{RM}$ squat test $\left(\mathrm{r}=0.68, \mathrm{r}^{2}=0.47, \mathrm{p}=0.0014\right)$ at the pre-test and post-test $\left(\mathrm{r}=0.88, \mathrm{r}^{2}=0.77, \mathrm{p}=0.001\right)$. When comparing the coefficients of correlation $(\mathrm{r}=0.68$ and $\mathrm{r}=0.88)$ between the squat tests and $\mathrm{VJ}$ tests, the difference that was noted was significant $(\mathrm{p}<0.001)$. A strong significant relationship was also noted between the 1RM squat and VJ test relative to body weight at pre-test $\left(\mathrm{r}=0.89, \mathrm{r}^{2}=0.79, \mathrm{p}=0.001\right)$ and post-test $\left.\left(\mathrm{r}=0.95, \mathrm{r}^{2}=0.90\right), \mathrm{p}<0.001\right)$. This study demonstrates that maximum strength in squats is a major predictive factor for jumping height in young female volleyball players. Female volleyball players might therefore consider focusing on maximum strength training to improve their jumping performance.
\end{abstract}

Keywords: Jumping performance, Correlation, 1 RM squat, Resistance training.

\section{INTRODUCTION}

The primary reason for resistance training in sports is strength and power improvements and athletic performance enhancement. It has been argued that enhancing and maintaining maximum strength is essential when considering the long-term development of power [1]. A variety of training methods are therefore used to increase strength and power in sports in order to enhance physical performance and thereby specific team sport performance, such as sprinting and jumping [2-4]. Prior studies have shown that maximum strength was able to determine sprint performance and jumping height in athletes $[5,6]$ and that throwing (ball) velocity correlates with strength performance in the upper extremities [7-10]. It has also been suggested that strength training could improve aerobic endurance performance, in the form of improved running economy, due to improvements in neuromuscular characteristics, including motor unit recruitment and reduced ground contact time [11-13].

Although previous studies have investigated the effect of maximum strength assessment and resistance training for several outcomes on power performance, it is important continually to examine this relationship to fully understand

*Address correspondence to this author at the Department of Food and Nutrition and Sport Science, Faculty of Education, University of Gothenburg, Box 300, SE 40530 Göteborg, Sweden; Tel: 0046(0)31 7862237; Fax: 0046(0)31 7862348;

E-mail: sofia.augustsson@gu.se its status in sport conditioning programs. Present researchhargues that resistance training might be an important factor in positively influencing jumping and throwing performances in highly trained female volleyball players [3]. However, few data exist on the relationship between maximum strength and power performance, such as jumping ability in low-level female volleyball players. There are still questions relating to the strength-power issue that remain to be answered. What is the relationship between power and muscular strength in lower extremity in non-elite female volleyball players? To what extent is the jumping ability depending on muscular strength in those athletes? What is the effect of resistance training on the relationship between muscular strength and jumping performance? The hypothesis in the current study is that there is a relatively strong relationship between maximal muscular strength and power also in young low-level female volleyball players and that resistance training leads to increased muscular strength and power. The aim of the present study was to determine the relationship between maximum strength and jumping performance in young female non-elite volleyball players before and after an in-season resistance-training protocol.

\section{MATERIALS AND METHODS}

The present research examined the changes in performance measurements of strength and power in ten young female volleyball players who participated in a 26 week, in-season resistance-training program. 
Table 1. Subject Characteristics $(n=10)$ at the Pre-Test. Values are Means ( \pm Standard Deviation)

\begin{tabular}{|c|c|}
\hline Age $(\mathbf{y})$ & $\mathbf{1 9}( \pm \mathbf{2})$ \\
\hline \hline Weight $(\mathrm{kg})$ & $66( \pm 19)$ \\
\hline Height $(\mathrm{cm})$ & $171( \pm 7)$ \\
\hline
\end{tabular}

\section{Subject}

Ten female volleyball players from the third division, Göteborg volley ball federation, Sweden, were invited to participate in the study. The players in this study have been involved in a larger study analyzing the effects of a 26 -week, individualized and supervised strength and injury prevention program focusing on performance enhancement in young female volleyball players who completed resistance training with either a supervised, individualized training program or an unsupervised, non-individualized training program [14, 15]. All players from the experimental group $(n=10)$, performing the supervised and individualized training program, were selected from the previous intervention study. None of the players had previous experience of resistance training, but they had previously performed conditioning training instructed by the main coach (e.g. plyometrics, body-weight strengthening exercises). The players took part in normal volleyball training twice a week during the season. No other conditioning training was performed, except for the intervention protocol, during the study period.

Written information was given to each player and written informed consent was obtained. Ethical approval was obtained from the Human Ethics Committee at Gothenburg University, Sweden. The players' age, height and weight were documented, as shown in Table 1. The players' weights were documented twice, at pre-test and at post-test.

\section{Procedure}

All the players were tested for physical performance, on a first occasion (pre-test) during the pre-season and one week prior to the initiation of the intervention. The intervention started after the pre-test and lasted for the whole of the 26week intervention season. All players received a program of resistance-training exercises that was designed by the first author and aimed to improve physical performance. All players were tested on a second occasion (post-test)in April 2008, after the end of the intervention season.

The 1 RM squattest was used to evaluate the players' maximum strength in the lower extremities and a vertical jump (VJ) test was used to assess functional performance and power. All players were given instructions relating to the test procedures in writing two weeks before the pre-test. The tests were performed in the same order on both test occasions. The players were also tested at the same time of the day, in the morning. The players were given strong verbal encouragement to perform all the tests as well as they could. Each test session began with a warm-up, which consisted of five minutes of ergo meter cycling at 100 Wof resistance. The tests were instructed and supervised by two test leaders with previously experience of physical strength and power testing.

\section{Measurements}

\section{Vertical Jump}

The vertical jump test (VJ) was performed as a countermovement jump, which has previously been described by Gustavsson et al. [16]. The players performed the jump from anup right and extended leg position with their hands placed on their waist. The players quicklybent their knees and then immediately jumped upwards for maximum height. Acomputerized system (Muscle Lab, Ergo test Technology) using a field of infrared light (approximately $10 \mathrm{~mm}$ above the floor), serving as a contact mat, made it possible to measure the flight time. The system then converted the flight time into jump height in centimeters. The players were instructed to jump upwards and the test leader registered the landing position. Failure to do so resulted in a disqualified hop. The players were tested to the point at which no further improvement was made, 3-10 trials. The best attempt was used for further analysis. High test-retest reliability (ICC $=0.95$ ) has previously been reported with this testing approach [16].

\section{RM squat}

The 1 RM squat test began with a standing back extension warm-up exercise consisting of 20 repetitions with no extra load. Further, 20 repetitions of the squat exercise were performed with no extra load. Before the test session, adjustment, barbell placement on the rack, was made to fit the player's height. For the test, the player dismounted bar from rack and stood in an upright starting position, in a safety squat rack, with an Olympic bar bell, which was the starting weight for all the players, on her shoulders placed high on the trapezius muscle. The player's feet were shoulder width apart, with the chest up and the eyes fixed straight ahead. The player performed the squat by descending to a parallel squat position, approximately $110^{\circ}$ of knee flexion, by bending her knees and hips until the greater trochanter of the femur reached the same horizontal plane as the superior border of the patella. The player then ascended to the starting position following a verbal signal from the test leader. A board ( $2 \mathrm{~cm}$ thick) elevating the heels of the player was used, thereby facilitating the parallel squat position. In addition, a tight weight belt supporting the trunk was obligatory. The player was instructed to be as upright as possible. A mirror placed $1.5 \mathrm{~m}$ in front of the player enabled visual feedback. The test leader stood behind the player, with his arms placed around the waist of the player during the lift to secure the exercise and to ensure that the proper form and technique were maintained. Any trials failing to meet the standardized technique criteria were discarded. The weight lifted for each trial was increased by $5-10 \mathrm{~kg}$ until failure occurred. The amount of load that was added before every attempt was based on the player's estimated perceived effort of the previous attempt together with the test leader's evaluation of the lift performance. The test leader was responsible for the loading of every trial. 1 RM was performed using one-minute resting periods between trials. For the post-test, the bar was loaded to $75 \%$ of the 1 RM performances from the pre-test as the starting load. High reliability has been noted for this testing protocol of the $1 \mathrm{RM}$ squat test $\left(\mathrm{ICC}_{2,1}=0.85\right),[17]$. 


\section{Resistance-Training Protocol}

The training program consisted of 26 weeks of progressive resistance training divided into three phases, familiarization phase, progression phase 1 and progression phase 2 . The programs were individualized and supervised [14]. A physical therapist supervised the players and was responsible for ensuring that exercise prescriptions were carried out correctly and were achieved during a particular workout (e.g. velocity of movement, appropriate spotting and technique, as well as safety considerations, intensity of the training). The resistancetraining exercises for the lower extremities were seated knee extension, lying leg curl, leg press, machine seated calf raises, body-weight one-leg squat and barbell squat. The players performed all the squat training in a free weight using a standard Olympic 20-kg barbell. The program was periodized, starting with a four-week familiarization phase with one training session per week. During this phase, the players performed exercises with approximately $70 \%$ of 1 RM. During the familiarization phase, the players focused on the correct technique and form and adaptation of the load. After the familiarization phase, progression phase 1 was initiated. During the following 10 weeks, the aim of the training was progression with one training session per week. The training load was approximately $80 \%$ of $1 \mathrm{RM}$ and was adjusted, if possible, every two weeks to maintain the $80 \%$ level. After progression phase 1, progression phase 2 was introduced and consisted of 12 weeks of high-intensity resistance training, with two training sessions per week, focusing onmaximum strength and performance. The training intensity was increased to $90-100 \%$ of $1 \mathrm{RM}$ in the squat exercise. Using standard trial and error methods (i.e., unload if too few repetitions were performed and load if too many repetitions were performed), the loads for each set were modified based on performance with reference to the target $1 \mathrm{RM}$. The physical therapist was responsible for this careful modulation of the training load progression. In addition, exercise testing was carried out by the physical therapist. More detailed information about the program can be found elsewhere $[14,15]$.

\section{Statistical Analyses}

The data are presented as the mean (SD). Pre- and postintervention data were analyzed with Wilcoxon's signedrank test. The relationships between the $1 \mathrm{RM}$ squat test and the VJ test were investigated, using Pearson's correlation coefficient. The relationship between the relative $1 \mathrm{RM}$ squat (1 RM/body weight) and relative vertical jump height (RVJ=vertical jump height/body weight) was also investigated. The difference between pre- and postcorrelation coefficients was examined using Hotelling's Tsquare test. The effect size was computed to express the difference in means in terms of standard deviations, with the pooled standard deviation according to Cohen's d [18]. Calculations prior to the study, using a power of $80 \%$, showed that pre- and post-intervention changes of at least 2 $\mathrm{cm}$ in jumping height and $10 \mathrm{~kg}$ in $1 \mathrm{RM}$ squat performance were needed to detect a significant effect of the intervention program with the ten players.

\section{RESULTS}

All the players $(n=10)$ completed the resistance-training program during the entire season, as well as the pre- and post-test sessions.

\section{Physical Performance}

The maximum weight lifted in the $1 \mathrm{RM}$ squat at pre-test was $41.5 \mathrm{~kg}(\mathrm{SD} \pm 14)$ and, for the post-test, after the intervention, $70 \mathrm{~kg}(\mathrm{SD} \pm 12)$. There was a significant difference between the two test sessions $(p=0.005)$, with an enhancement of $69 \%$. The effect size was 2.16 , with a CI of 1.5-2.9.

The maximum height measured for the VJ test at pre-test was $25.9 \mathrm{~cm}(\mathrm{SD} \pm 4)$ and for the post-test, after the intervention, $28.3 \mathrm{~cm}(\mathrm{SD} \pm 4)$. A significant difference was noted between the two test sessions $(p=0.008)$, with an enhancement of $9 \%$. The effect size was 0.58 , with a CI of 0.28-0.88.

\section{Relationship between Muscular Strength and Functional Performance Tests}

A strong significant relationship was found between the $\mathrm{VJ}$ test and the $1 \mathrm{RM}$ squat test $(\mathrm{r}=0.68, \mathrm{r} 2=0.47, \mathrm{p}=0.0014)$ at the pre-test and the post-test $(\mathrm{r}=0.88, \mathrm{r} 2=0.77, \mathrm{p}=0.001)$. When comparing the coefficients of correlation $(\mathrm{r}=0.68$ and $\mathrm{r}=0.88$ ) between the squat tests and VJ tests, the difference that was noted was significant $(p<0.001)$. Fig. (1) illustrates the correlations between the $1 \mathrm{RM}$ squat test and the $\mathrm{VJ}$ at pre-test, whereas Fig. (2) illustrates the correlations between the 1 RM squat test and the VJ at post-test. A strong significant relationship was also noted between the relative $1 \mathrm{RM}$ squat and RVJ at pre-test $(\mathrm{r}=0.89, \mathrm{r} 2=0.79, \mathrm{p}=0.001)$ and at post-test $(\mathrm{r}=0.95, \mathrm{r} 2=0.90), \mathrm{p}<0.001)$. There was no significant difference between the players' pre-test weight, $66 \mathrm{~kg}$, and post-test weight, $67 \mathrm{~kg}(\mathrm{p}=0.343)$.

\section{DISCUSSION}

This study provides additional support for previous research $(5,6)$ on the relationship between maximum strength and power. In the present study, the correlations between the $1 \mathrm{RM}$ squat and $\mathrm{VJ}$ were found to be high $(\mathrm{r}=0.68$ and $\mathrm{r}=0.88$ ). Interestingly, the correlation was improved with the enhancement of strength performance. Further, the correlation relative to body weight at post-test was found to be extremely high $(\mathrm{r}=0.95)$. This result suggests that $90 \%$ of the vertical jump performance in the present study is a result of maximum squat strength $(\mathrm{r} 2=0.90)$. The outcome of the present study therefore suggests that muscle strength plays a major and important role when it comes to performance in power exercises. This is in accordance with a previous study in which the 1RM squat test, expressed relative to body mass, was shown to have a high correlation with vertical jump performance [5]. However, the mechanisms driving these improvements and contributing to the relationship between power and maximum strength could not be explained from the present investigation. It might be that the players changed their jumping pattern (i.e. used more muscle strength than jumping technique), or increased their rate of force development, or changed their muscle architecture. The correlations can only give an insight into associations and we can only speculate on the cause and effect in this study. In spite of this, it is still possible to state from the current study that the increase in squat performance definitely contributed to the improved jumping performances. Moreover, based on the coefficient of determination, in the present study, the 


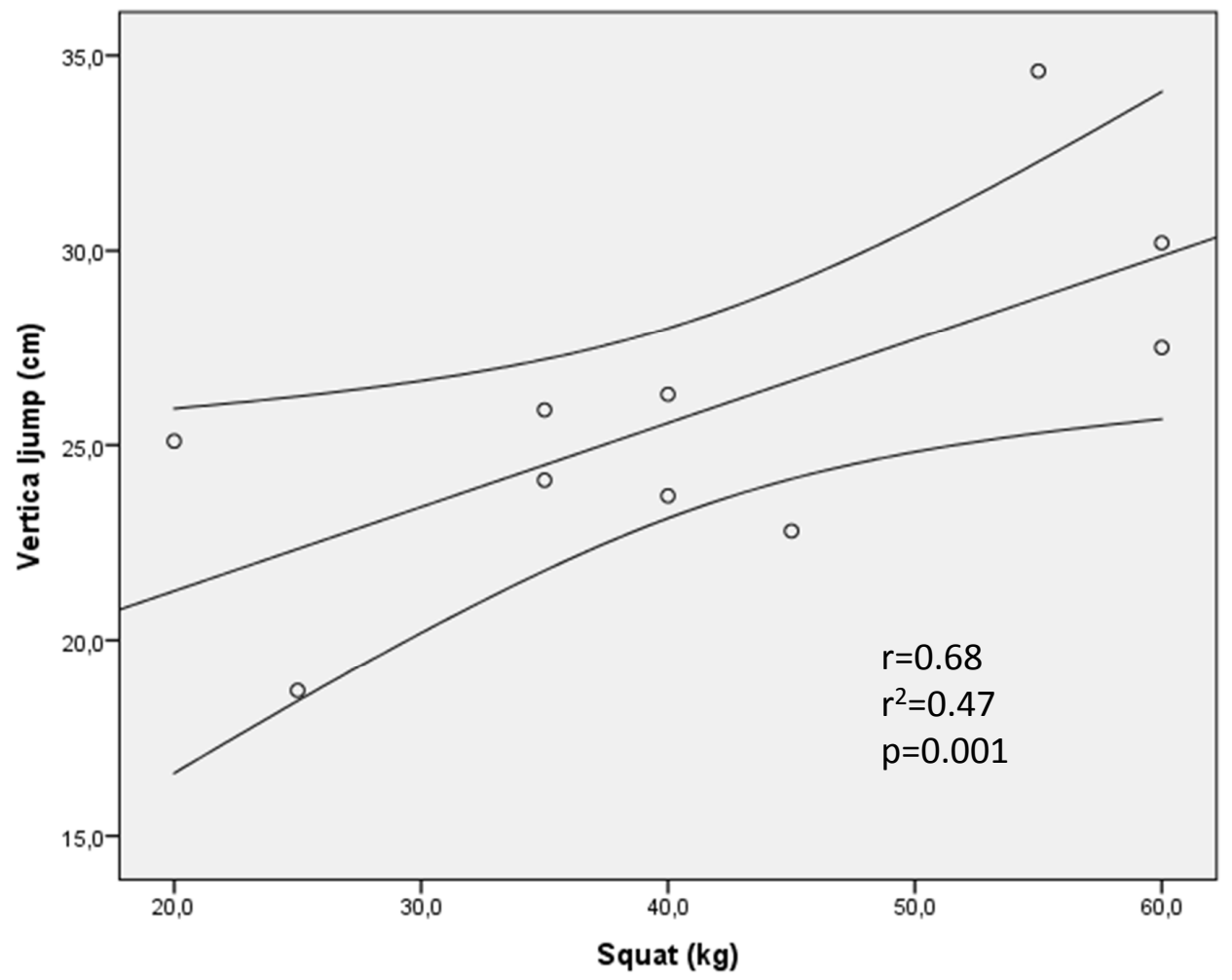

Fig. (1). Correlation between the vertical jump test and the 1 RM squat test, in young female volleyball players ( $\mathrm{n}=10)$, at pre-test with $95 \%$ confidence interval.

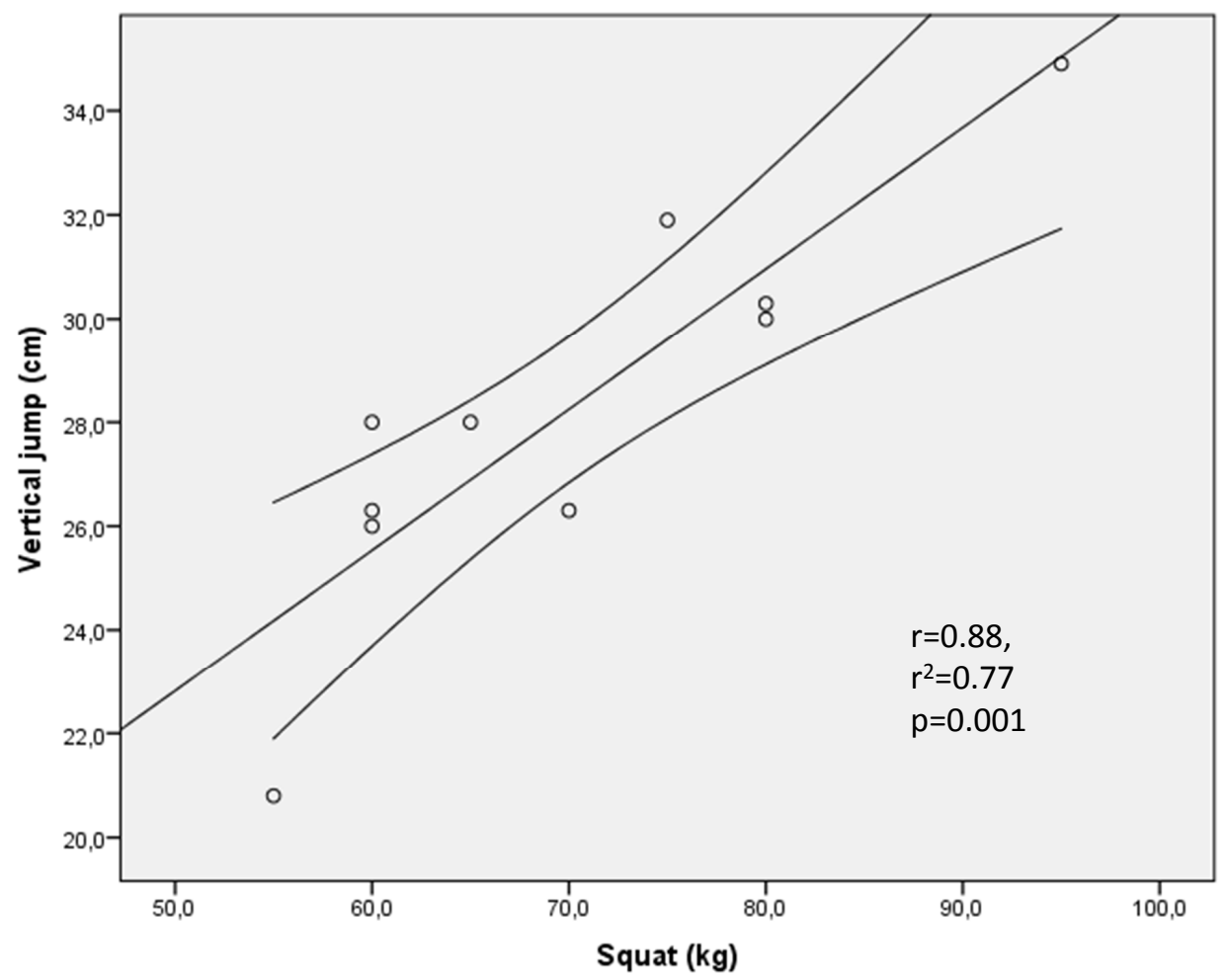

Fig. (2). Correlation between the vertical jump test and the 1 RM squat test, in young female volleyball players ( $\mathrm{n}=10$ ), at post-test after 26 weeks of resistance training with $95 \%$ confidence interval.

maximum squat strength appears to be a key predictive factor for jumping height in young female volleyball players.
This study suggests that female volleyball players can improve their strength and power during the competition 
season by applying a resistance-training program. The pre/post differences for the squat and vertical jump test were $67 \%$ and $9 \%$ respectively, which most likely represent practically meaningful improvements. Not surprisingly, the size of the effect of the resistance-training program on maximum strength (2.16) was large. The size of the effect on jumping performance (0.58) was intermediate. Interestingly, the effect on jumping ability in the present study is similar to the outcome after plyometric exercises [19]. However, since jumping is one of the most important tasks in volleyball, it is most likely that this ability will improve during one season of volleyball training. As a result, the size of the effect on jumping performance in the present study could be partly due to the normal adaptation to sports participation.

Previously, few studies have examined the effect of entire in-season resistance training on physical performance in sport. Designing an ideal resistance-training program for sport can be a complex process in itself. It is often recommended that in-season training programs should aim to maintain the muscular strength and power developed during the off-season. One main concern, when it comes to inseason strength and conditioning programs, is the risk of their conflicting with the competition preparations and the high training volume they entail. Research has suggested that the training load needs to be carefully monitored throughout an in-season period to maintain optimal neuromuscular performance throughout an athlete's entire sporting season [20]. However, the findings in the current study suggest that a relatively high-intensity, in-season resistance-training program does not impair the effect on specific sporting performance. In fact, the in-season, resistance-training program in the present study could be recommended to enhance sporting performance such as jumping ability. It is, however, important to remember that the sample in the present study consisted of low-level volleyball players and the effect on volleyball players in higher divisions cannot be assessed. The training volume in elite volleyball players is much higher and, for this reason, the resistance-training regimen (e.g. volume, frequency and intensity) is probably dissimilar. The resistance training prescribed for the players in the present study was periodized. Since the athletes in the present study were inexperienced in resistance training, we set the starting load at $70 \%$ of $1 \mathrm{RM}$, in order gradually to begin learning the correct technique. However, the more advanced the athletes become in performing the exercises, the more variation (i.e. specific training cycles) may be necessary to avoid performance plateaus [21]. For this purpose, the training load was increased to approximately $80 \%$ of $1 \mathrm{RM}$ (progression phase 1), after the familiarization phase and, further, to $90-100 \%$ of $1 \mathrm{RM}$ in progression phase 2 , to enhance progression. The intensity of the resistance training was therefore at its highest at the end of the competition season.

The 1 RM test in the present study were performed using one-minute resting periods between trials. Research has indicated that the rest interval between sets is an significant variable that affects both acute responses and chronic adaptations to resistance training programs [22, 23]. Previous studies has shown that 1-minute rest intervals are sufficient for recovery between attempted lifts during 1RM testing for the free-weight back squat test [24]. Thus, we used the one-minute resting periods between trials in the current study.

\section{METHODOLOGICAL ISSUES}

The primary limitation of this research is the absence of a control group. Due to the present study design, we could not compare the effect of this particular training program to other interventions or to a control group. Also, the values from the present study could only be applied to young novice lifters. Thus, precautions should be taken into account when generalizing to other more experienced lifters. Yet, these data were intended to describe the relationship between maximum strength and jumping performanceand not to evaluate a specific training regime. The effect of this specific program has previously been investigated with a control group and the result can be found elsewhere [14]. Moreover, we suspect that neither of the limitationsaffects the primary result of this study, that there is a strong relationship between maximum strength and jumping performance in young female volleyball players.

\section{CONCLUSIONS}

This study demonstrates that maximum strength in squats is a major predictive factor for jumping height in young female volleyball players. The results from the present study suggest that female volleyball players should consider focusing on maximum strength training to improve their jumping performance. The data from the present study also support the use of resistance training for in-season conditioning.

\section{CONFLICT OF INTEREST}

The authors confirm that this article content has no conflicts of interest.

\section{ACKNOWLEDGEMENT}

This study was supported by a grant from Swedish National Center for Research in Sports.

\section{REFERENCES}

[1] Cormie P, McGuigan MR, Newton RU. Developing maximal neuromuscular power: part 2 - training considerations for improving maximal power production. Sports Med 2011; 41: 12546.

[2] Gabbett TJ, Johns J, Riemann M. Performance changes following training in junior rugby league players. J Strength Cond Res 2008; 22: $910-7$.

[3] Marques MC, Tillaar R, Vescovi JD, González-BadilloJJ. Changes in strength and power performance in elite senior female professional volleyball players during the in-season: a case study. J Strength Cond Res 2008; 22: 1147-55.

[4] Santos EJ, Janeira MA. Effects of complex training on explosive strength in adolescent male basketball players.J Strength Cond Res 2008; 22: 903-9.

[5] Nuzzo JL, McBride JM, Cormie P, McCaulley GO. Relationship between countermovement jump performance and multijoint isometric and dynamic tests of strength. J Strength Cond Res 2008; 22: 699-707

[6] Wisløff U, Castagna C, Helgerud J, Jones R, Hoff J. Strong Correlation of Maximal Squat Strength with Sprint Performance and Vertical Jump Height in Elite Soccer Players. Br J Sports Med 2004; 38: 285-8.

[7] Forthomme B, Croisier JL, Ciccarone G, Crielaard JM, Cloes M. Factors correlated with volleyball spike velocity. Am J Sports Med 2005; 33: 1513-9. 
[8] Gorostiaga EM, Granados C, Ibáñez J, Izquierdo M. Differences in physical fitness and throwing velocity among elite and amateur male handball players. Int J Sports Med 2005; 26: 225-32.

[9] Marques MC, van den Tilaar R, Vescovi JD, Gonzalez-Badillo JJ. Relationship between throwing velocity, muscle power, and bar velocity during bench press in elite handball players.Int $\mathrm{J}$ Sports Physiol Perform 2007; 2: 414-22.

[10] Prokopy MP, Ingersoll CD, Nordenschild E, Katch FI, Gaesser GA, Weltman A. Closed kinetic chain upper-body training improves throwing performance of NCAA Division I softball players. J Strength Cond Res 2008; 22: 1790-8.

[11] Hoff J, Gran A, Helgerud J. Maximal strength training improves aerobic endurance performance. Scand J Med Sci Sports 2002; 12: 288-95.

[12] Hoff J, Helgerud J, Wisløff U. Maximal strength training improves work economy in trained female cross-country skiers. Med Sci Sports Exerc 1999; 31: 870-7.

[13] Jung AP. The impact of resistance training on distance running performance. Sports Med 2003; 33: 539-52.

[14] Augustsson SR, Augustsson J, Thomeé R, Karlsson J, Eriksson BI, Svantesson U. Performance enhancement following a strength and injury prevention program: a 26-week individualized and supervised intervention in adolescent female volleyball players. J Sports Sci Coach 2011; 3: 399-417.

[15] Augustsson SR. Strength training for physical performance and injury prevention in sports. Individualised and supervised training for female athletes. Ph.D. dissertation. Sweden Institute of Neuroscience and Physiology, The Sahlgrenska Academy at
Göteborg University 2009. Available at: http:/hdl.handle.net/2077/20448.

[16] Gustavsson A, Neeter C, Thomee P, et al. J. Test Battery for Evaluating Hop Performance in Patients with an ACL Injury and Patients Who Have Undergone ACL Reconstruction. Knee Surg Sports Traumatol Arthrosc 2006; 14: 778-88.

[17] Augustsson SR, Svantesson U. Reliability of the 1 RM bench press and squat in young women. Eur J Physiother 2013; Early Online: 19.

[18] Cohen J. Statistical Power Analysis for the Behavioral Sciences. $2^{\text {nd }}$ ed. New Jersey: Lawrence Erlbaum Associates 1988.

[19] Markovic G. Does plyometric training improve vertical jump height? A meta-analytical review.Br J Sports Med 2007; 41: 34955.

[20] McLean BD, Petrucelli C, Coyle EF. Maximal power output and perceptual fatigue responses during a division I female collegiate soccer season.J Strength Cond Res 2012; 26: 3189-96.

[21] Kraemer WJ, Ratamess NA. Fundamentals of Resistance Training: Progression and Exercise Prescription. Med Sci Sports Exerc 2004; 36: 674-88.

[22] Willardson JM, Burkett LN. The effect of rest interval length on bench press performance with heavy vs. light loads. J Strength Cond Res 2006;20: 396-9.

[23] Senna G, Willardson JM, de Salles BF, et al. The effect of rest interval length on multi and single-joint exercise performance and perceived exertion.J Strength Cond Res 2011; 25: 3157-62.

[24] Matuszak ME, Fry AC, Weiss LW, Ireland TR, McKnight MM. Effect of rest interval length on repeated 1 repetition maximum back squats. J Strength Cond Res 2003; 17: 634-7.

(c) Sofia Ryman Augustsson; Licensee Bentham Open.

This is an open access article licensed under the terms of the Creative Commons Attribution Non-Commercial License (http://creativecommons.org/licenses/ by-nc/3.0/) which permits unrestricted, non-commercial use, distribution and reproduction in any medium, provided the work is properly cited. 